\title{
The effect of auditory hallucinations on loudness of verbal report
} CHARLES G. STEWART, Tulane University. New Orleans,
La. 70118

The loudness of verbal reports by three very hypnotizable, highly trained Ss were rated during both hypnotic auditory hallucinations and real sound changes. Loudness of roice varied significantly as a function of actual sound level but apparently not at all as a function of hallucinated level. This result accords with the "skeptical" position on hallucinations and other hypnotic phenomena and, in particular, with the evidence from studies of auditory hallucinations involving suppression of previously conditioned responses or of the disruptive effects of delayed auditory feedback.

Recent hypnotic research supports what Sutcliffe (1961) has termed the "skeptical" view: that hypnotic suggestions do not instigate organismic processes identical or even closely similar to those produced by the corresponding actual conditions and stimuli. Hypnotic auditory hallucinations, for example, do not match real auditory stimuli in their behavioral effects. Barber (1961) reviewed studies which demonstrated that suggested unilateral deafness breaks down when sounds are presented in such a way that Ss can not determine which ear is being stimulated and that hypnotic bilateral deafness does not prevent speech impairment during delayed auditory feedback. Barber \& Calverley (1964) found that suggested deafness with or without a prior hypnotic induction evoked significantly more subjective reports of partial or complete deafness than occurred in a control group receiving no suggestions. Yet none of the three groups differed in any important respect on the disruptive effects of delayed auditory feedback on speech. Sutcliffe (1961) used arithmetic problems whose steps and solutions were required to be spoken aloud to test not only the effects of negative hallucinations (deafness) but also the effects of positive hallucinations with respect to delayed auditory feedback. One of several conditions involved hypnotic deafness for ongoing delayed feedback and another condition consisted of hallucinated voice delay when in fact there was none. Except for a decremental influence of the trance state itself on highly susceptible Ss, performance impairment was a function entirely of the actual presence or absence of delayed feedback.

Somewhat less stringent tests of hypnotic hallucinations have appeared to offer more encouraging behavioral evidence. Barber (1961, 1965) has reviewed a number of studies in which hypnotic deafness seemed to suppress startle-responses to loud sounds or classically-conditioned responses to acoustic stimuli. However, in each case, an interpretation of voluntary suppression is possible-e.g., covert muscle potential changes to startle-stimuli. And as Barber (1961) pointed out, there also is evidence that nonhypnotized $\mathrm{Ss}$ can voluntarily inhibit conditioned responses.

Despite the failure of hallucinations to replicate actual sensory effects, the persistence of Ss' reports of genuine perceptual experience leads many researchers to assume subject sincerity rather than simulation during hypnotic hallucinations. As Sutcliffe (1961) has remarked, the skeptical viewpoint can encompass both the simulation and delusion interpretations of subjective reports accompanying hypnotic behavior. Although objective evidence, apart from verbal report, of such delusion is hard to attain, it is possible that a behavioral index not involving suppression of other responses might reflect Ss' honest beliefs under certain circumstances. During the course of other experimental work involving tachistoscopic identifications during positive and negative auditory hallucinations (Stewart, 1965), E thought he detected a tendency for the loudness of Ss' verbal identifications to correlate with hallucinated as well as real auditory loudness. Such behavior would be an outgrowth of the obvious fact that most people automatically raise or lower their voices to match the background level of noise.

\section{METHOD}

Three University of Michigan undergraduates originally selected from volunteers for paid research mainly on the basis of high scores (11 or 12 out of 12) on Form $A$ of the Stanford Hypnotic Susceptibility Scale (Weitzenhoffer \& Hilgard, 1959) served in the study. These Ss had been thoroughly trained in hypnotic procedures, amnesia, hallucinations, and other experiences for participation in an extensive series of experiments. In particular they had learned to hallucinate increases and decreases in sound intensity in response to posthypnotic cues presented in the adapting field of a Gerbrands tachistoscope. The sound was white noise delivered through earphones. The hallucination training had been careful and intensive to ensure good hallucinations and amnesia for the difference between real and hallucinated sound changes. The details of this rigorous training can be found in Stewart (1965).

Two sound levels were employed in this study: a white noise of $87 \mathrm{~dB}$ played through earphones from a tape recorder and the residual tape recorder hum of $62 \mathrm{~dB}$ when the gain control was turned down. Actual sound changes were affected gradually by a continuous turn of the gain control, and hallucinated changes occurred at the same pace. Posthypnotic cues comprised a symbol-either a square for real sound manipulation or a check for hallucinated level-and a number designating which of the two sound levels would occur. Ten types of trials were formed. five beginning with each of the two sound levels. A square plus the number indicating the same level that began the trial meant no sound change (62-62 or 87-87); a square with the number of the other sound meant that a real change controlled by $E$ would occur $(62-87$ or $87-62)$. A check plus the number of the same sound level meant $\mathrm{S}$ would hear (by hallucination) that same sound throughout the trial regardless of what happened; some times no actual change occurred $(62-\mathrm{H} 62$ or $87-\mathrm{H} 87)$, but on other trials $E$ shifted the real sound to the opposite level [62-H62(87) or $87-\mathrm{H} 87(62)]$, a change which $\mathrm{S}$ did not consciously hear because of the hallucination. Finally a check accompanied by the number of the opposite sound level indicated a direct hallucinatory increase $(62-\mathrm{H} 87)$ or decrease (87-H62). These 10 trials occurred once in a simple sequence which was then repeated in the reverse order, making 20 trials in all.

Each trial began with $S$ looking at whatever posthypnotic cue had been placed in the adapting field of a Gerbrands tachistoscope. When the cue was removed, $\mathbf{S}$ closed his eyes. As soon as any indicated sound changes had reached the appropriate level, $S$ pressed the flash button activating the tachistoscopic timer. If no sound change was supposed to occur, $S$ delayed his press an equivalent interval. The $S s$ were required to judge when the intensity level was appropriate by pressing the button themselves in order to indicate to $E$ the intensity of Ss' hallucinations. The investigator could then observe how closely each $S$ approximated the real training sound of $87 \mathrm{~dB}$ on trials involving actual sound changes. (S3 tended to overshoot into the region of $90 \mathrm{~dB}$.) At the moment during the noise from the timer sequence when the stimulus flash would ordinarily occur, the mental image of a dark letter $X$ against a white background 
Table 1

Frequencies of Voice Loudness Ratings

\begin{tabular}{|c|c|c|c|c|}
\hline \multirow{2}{*}{$\begin{array}{l}\text { Loudness } \\
\text { Category }\end{array}$} & \multicolumn{4}{|c|}{ Trial category } \\
\hline & $\begin{array}{l}\text { Loud objectively } \\
\text { Loud subjectively }\end{array}$ & $\begin{array}{l}\text { Loud objectively } \\
\text { Soft subjectively }\end{array}$ & $\begin{array}{l}\text { Soft objectively } \\
\text { Loud subjectively }\end{array}$ & $\begin{array}{r}\text { Soft objectively } \\
\text { Soft subjectively }\end{array}$ \\
\hline \multicolumn{5}{|c|}{ Subject 1} \\
\hline Loud & $3(3)$ & $3(4)$ & $0(0)$ & $0(0)$ \\
\hline Medium/Soft & $3(3)$ & $1(0)$ & $4(4)$ & $6(6)$ \\
\hline \multicolumn{5}{|c|}{ Subject 2} \\
\hline Loud & $6(5)$ & $4(3)$ & $0(0)$ & $0(0)$ \\
\hline Medium/Soft & $0(1)$ & $0(1)$ & $4(4)$ & $6(6)$ \\
\hline \multicolumn{5}{|c|}{ Subject 3} \\
\hline Loud & $6(6)$ & $4(4)$ & $0(0)$ & $0(0)$ \\
\hline Medium/Soft & $0(0)$ & $0(0)$ & $4(4)$ & $6(6)$ \\
\hline
\end{tabular}

Note-Ratings by the experimental assistant are given in parentheses.

entered S's mind. Immediately S reported how black the $\mathrm{X}$ had been, using a prearranged code not relevant to this study. Two Es, the principal $\mathrm{E}$ who knew what was happening on each trial and an assistant who did not, rated the loudness of each verbal response as "loud," "medium," or "soft."

RESULTS AND DISCUSSION

Since the category "loud" was used by both raters almost as frequently as the other two categories together, these latter were combined. Table 1 presents the data for both raters on all three Ss. As the table indicates, the 10 types of trials can be partitioned into four categories according to whether they involved objectively loud or soft sounds and whether they would appear loud or soft subjectively to Ss undergoing hallucinations during the verbal response. Trials $87-87,62-87$, and $87-\mathrm{H} 87$ were loud both objectively and subjectively. Trials $62-62,87-62$, and 62-H62 were soft from both viewpoints. The divergence occurred on the other four typès of trial. Trials $87-\mathrm{H} 62$ and 62-H62(87) were subjectively soft but objectively loud. And 62-H87 and 87-H87(62) should have been subjectively loud during the verbal response but were objectively soft. Since each trial was repeated twice, the frequencies are doubled.

A cursory bunce suffices to suggest that voice loudness was a function of actual intensity of sound during verbal report and not of hallucinated loudness. When the data are grouped into 2-by-2 tables for each $S$ and each rater according to objective loudness and softness and separately according to subjective sound level, the resulting six tables for objective sound level all yield $\chi^{2}$ 's significant beyond the .01 level whereas the resulting six tables for subjective levels all fail to yield probability values as high as the .25 level. The rather simple estimates of loudness by the two raters appear to have been sufficient considering the close agreement in frequencies. It should be emphasized that the assistant $E$ was rating in complete ignorance and that the result came out counter to the principal E's expectations.

These results fit well with the experimental data reviewed above and also accord with the general supposition that hypnotic Ss must know at some mental level the difference between reality and fantasy. The fact that amnesia for such a difference can be so readily removed indicates that such knowledge is always present somewhere below the top level of awareness. However, retrievability from memory need not imply current control over all response processes. Therefore, the possibility exists for $\mathrm{S}$ consciously feeling the need to match his voice level to his hallucinations when they are not recognized consciously as fantasy. Conceivably, E's initial impression was not totally incorrect but rather the original effect dissipated as the Ss grew more practiced. In other words, as they subconsciously learned to anticipate the experimental routine their deluded awareness may have exerted decreasing control over their actual behavior. This possibility could be evaluated by testing for such effects early in training rather than after the training was completed.

\section{REFERENCES}

BARBER, T. X. Physiological effects of "hypnosis." Psychological Bulletin, 1961, 58, 390-419.

BARBER T X Physiological effects of "hypnotic suggestions": A critical review of recent research $(1960-1964)$. Psychological Bulletin, $1965,63,201-222$.

BARBER, T. X., \& CALVERLEY, D. S. Experimental studies in "hypnotic" behavior: Suggested deafness evaluated by delayed auditory feedback. British Journal of Psychology, 1964, 55, 439-446.

SUTCLIFFE, J. P. "Credulous" and "skeptical" views of hypnotic phenomena: Experiments in esthesia, hallucination, and delusion. Journal of Abnormal \& Social Psychology, 1961, 61, 39-46.

STEWART, C. G. Real versus hypnotically hallucinated sensory interaction as a test for inhibition. Unpublished doctoral dissertation, University of Michigan 1965.

WEITZENHOFFER, A. M., \& HILGARD, E. R. Stanford hypnotic susceptibility scale, forms $A$ and $B$. Palo Alto, Calif.: Consulting Psychologists Press, 1959.

NOTE

1. The data reported here were gathered at the University of Michigan as part of a group of studies funded by a Rackham Graduate Student Research Grant. Thanks are due to Maaja Agur Stewart for assistance in carrying out the experiment. 